\title{
Multi-dimensional differential imaging with FE-CARS microscopy
}

\author{
Vishnu Vardhan Krishnamachari, Eric Olaf Potma* \\ Department of Chemistry, Beckman Laser Institute, University of California Irvine, Irvine, CA 92697, USA
}

A R T I C L E I N F O

Article history:

Received 7 February 2008

Accepted 7 July 2008

Available online $\mathrm{xxx}$

\section{Keywords:}

Coherent Raman spectroscopy

CARS microscopy

Focus-engineering

Chemical interfaces

\begin{abstract}
A B S T R A C T
Focus-engineered coherent anti-Stokes Raman scattering (FE-CARS) microscopy is used to visualize microscopic samples with differential imaging contrast in both longitudinal and lateral directions. Multidimensional contrast is achieved by applying a one-dimensional $\pi$-phase step in the transverse beam profile of the Stokes beam. The observed longitudinal differential contrast results from fortuitous phasematching at axial interfaces and is highly sensitive to the resonant part of the third-order susceptibility. Based on phase-sensitive recordings of the vibrational spectrum of dimethyl sulfoxide in the $\mathrm{CH}_{3}$ stretching range, it is shown that FE-CARS uniquely probes the spectral phase of the Raman bands of the sample.
\end{abstract}

(c) 2008 Elsevier B.V. All rights reserved.

\section{Introduction}

Nonlinear coherent imaging techniques, such as secondharmonic generation (SHG) microscopy [1-4], third-harmonic generation (THG) microscopy [5-8] and coherent anti-Stokes Raman scattering (CARS) microscopy [9-11], provide high resolution images with contrast based on spatial variations in the second- and the third-order susceptibilities of the sample material. Besides providing useful endogenous contrast in biological imaging, these techniques share another attribute: the emitted signals are coherent.

Coherent emission implies that different points in the focal volume emit light with a very well-defined optical phase relative to one another. Hence the total signal detected is affected by the way the emitted waves interfere in the detector plane. When the emitted waves are in phase, the signal is strongest; on the other hand, the signal is substantially weaker if the waves are out of phase. While in some cases the coherent nature of these imaging techniques may complicate the interpretation and the analysis of the signals, the existence of coherence nonetheless provides a convenient handle towards emission control. If the optical phase of different parts of the focal volume can be administered, then the strength and the spatial shape of the phase-matched signal can be controlled. Such emission controllability would introduce new imaging capabilities for tuning several properties of the sample into sharpness.

\footnotetext{
* Corresponding author.

E-mail address: epotma@uci.edu (E.O. Potma).
}

One way to control the phase of the emitted wave from a given point in the focal volume is to manipulate the phase of the driving fields. Hence, through focus-engineering, i.e. phase shaping the incident radiation, coherent emission profiles can be directly controlled. When applied to coherent nonlinear microscopy, focusengineering offers several mechanisms for image contrast enhancement. This principle was recently applied to CARS microscopy and was shown to add a series of new contrast mechanisms to the existing palette of CARS microscope [12-15].

In contrast to SHG and THG microscopy, CARS is sensitive to molecular vibrational resonances. The detected CARS signal is a composite of the vibrationally resonant signal and a nonresonant electronic contribution, induced, respectively, by the resonant $\left(\chi_{\mathrm{r}}^{(3)}\right)$ and the nonresonant $\left(\chi_{\mathrm{nr}}^{(3)}\right)$ third-order susceptibilities of the material. In CARS microscopy, optimizing the vibrational contribution while suppressing the nonspecific nonresonant background is desirable yet challenging. To improve contrast in CARS images, the intrinsic differences between the phase properties of $\chi_{\mathrm{r}}^{(3)}$ and $\chi_{\mathrm{nr}}^{(3)}$ can be exploited. Because $\chi_{\mathrm{r}}^{(3)}$ is sensitive to vibrational resonances, it is complex, whereas $\chi_{n r}^{(3)}$ is real. This implies that the $\chi_{\mathrm{r}}^{(3)}$ response displays a phase dependence as a function of vibrational frequency, whereas the spectral phase of $\chi_{\mathrm{nr}}^{(3)}$ is flat throughout the vibrational spectral range of interest. Contrast improvement through CARS interferometry, for instance, is based on this phase difference [16-20]. Focus-engineered CARS (FE-CARS) also makes use of this phase difference to achieve image contrast enhancement. The improved vibrational contrast of FECARS microscope is obtained by suppressing the signal from the bulk medium through spatial destructive interference, while retaining the vibrationally resonant signal at interfaces. 
FE-CARS can be achieved by applying phase steps to the incident Stokes beam with a spatial light modulator [12,14]. For instance, by applying a $\pi$-phase step in $x$-direction across the transverse beam profile, the equivalent of the phase profile associated with a Hermite Gaussian (HG10) beam, the resulting focal CARS emission is negligible due to the engineered destructive interference of CARS waves generated in different parts of the excitation volume. However, the focal phase step can be compensated for by the spectral phase jump encountered at interfaces between vibrationally resonant and nonresonant objects in the sample under investigation. Indeed, it was recently demonstrated that FE-CARS with HG10 Stokes beams not only highlights interfaces of vibrationally resonant objects, but also suppresses the nonresonant background by more than an order of magnitude [14]. Such contrast enables differential imaging of vibrationally resonant objects, which is of particular relevance to cell culture imaging.

FE-CARS sensitivity to the orientation of an object's interface is intimately related to the kind of phase mask utilized. Calculations have shown that Stokes beams with HG10- and HG01-type phase profiles give rise to differential imaging in the lateral plane that are oriented parallel to $y$ - and $x$-axis, respectively [12]. Differential imaging in both directions can be achieved with a LaguerreGaussian Stokes beam profile [12], which is effectively a linear superposition of HG01 and HG10 modes [21]. Similarly, interfaces perpendicular to the longitudinal direction are efficiently highlighted when the Stokes beam resembles an 'optical bottle beam', which features an effective $\pi$-step along the propagation axis [13]. Nonetheless, for practical imaging applications it would be desirable to achieve differential contrast in both lateral and longitudinal directions using a single phase mask. In this contribution, we report on such multi-dimensional differential contrast using a simple HG10 phase profile for the Stokes beam. We explain the rather unexpected sensitivity of a transverse phase step to longitudinal interfaces in terms of fortuitous phasematching of the focal CARS waves in lateral and longitudinal directions. In addition, we emphasize that the observed multidimensional differential imaging contrast intimately correlates with the spectral phase of the probed Raman transition.

\section{Experimental}

A schematic of the FE-CARS setup is shown in Fig. 1. Our FECARS system is based on a $7 \mathrm{ps}, 1064 \mathrm{~nm}$ mode-locked laser (Picotrain, HighQ GmbH) which provides the Stokes beam, and a tunable $7 \mathrm{ps}$ optical parametric oscillator (Levante, APE) that delivers the pump beam for the CARS process in the $800-820 \mathrm{~nm}$ range. A liquid crystal spatial light modulator (SLM2256D, Meadowlark) is used to imprint a HG10 phase pattern onto the Stokes beam. The pump and Stokes beams are collinearly directed into a commercial laser scanning microscope (Fluoview 300, Olympus). Incident beams are focused with a high numerical aperture water immersion lens (1.15 NA, 40x, UApo, Olympus). Signal is collected in the forward direction with a $0.55 \mathrm{NA}$ condenser, spectrally filtered (650 nm bandpass, $40 \mathrm{~nm}$ bandwidth) and detected with a photomultiplier (R3896, Hamamatsu).

To determine the spectral phase of the CARS signal, our system is equipped with a CARS interferometer. Details of the CARS interferometer can be found elsewhere $[14,20]$ Briefly, the phase spectra are obtained by determining the phase shift between the CARS field from the Raman active sample and the nonresonant CARS field from glass by mixing each field with a sinusoidally modulated reference local oscillator beam. The recorded phase shift corresponds to the relative phase difference between the $\chi^{(3)}$ of the Raman active sample and the $\chi^{(3)}$ of the nonresonant glass. A

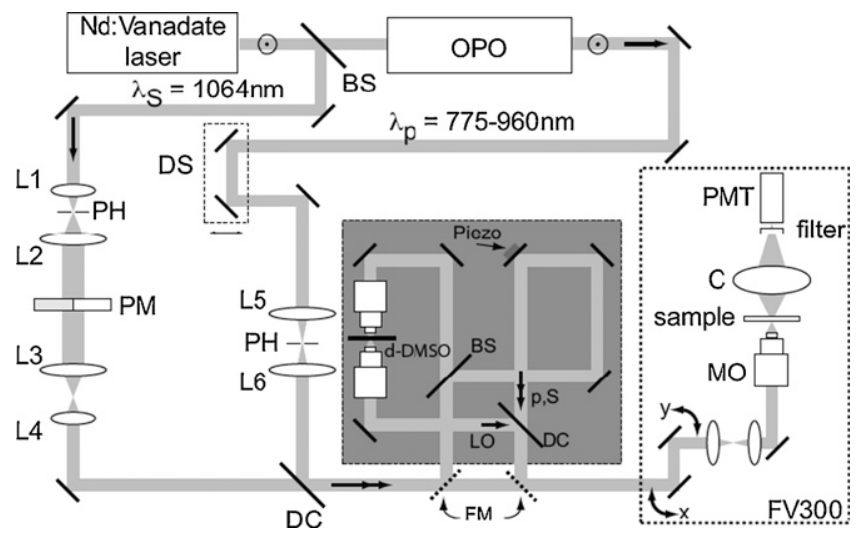

Fig. 1. Sketch of the experimental setup: OPO-optical parametric oscillator, L1L6-lenses, PH-pin-hole, PM-spatial light modulator, DS-delay stage DCdichroic, BS-beam-splitter, FM-flip mirror, MO-microscope objective, Ccondenser, PMT-photo-multiplier tube, p-pump beam, S-Stokes beam and LO-local oscillator field generated in a nonresonant sample of deuterated DMSO. Shaded region corresponds to the local-oscillator generator/CARS interferometer module employed to determine the spectral phase of the sample.

phase spectrum is acquired by determining the relative phase at different settings of the difference frequency between the pump and Stokes beams.

In addition, the microscope incorporates a Raman spectrometer. The $532 \mathrm{~nm}$ radiation $(\sim 10 \mathrm{~mW})$ split off from a frequencydoubled Nd:vanadate laser (Verdi V5, Coherent Inc.) is sent through the backport of the microscope frame and focused onto the sample with the same objective as used in CARS. Raman scattered light is filtered with a holographic notch filter (Kaiser) and detected through a grating spectrometer equipped with a cooled CCD camera.

To demonstrate multi-dimensional differential imaging contrast, we performed FE-CARS measurements on simple model systems. The sample used in this study is a layer of dimethyl sulfoxide (DMSO) diluted at a ratio of 1:5 in water, sandwiched between standard microscope coverslips. Small amounts of deuterated dodecane are added to the DMSO solution for forming D-dodecane micro-droplets that introduce microscopic contrast. In this work, the $\mathrm{CH}$ stretching region $\left(2800-3000 \mathrm{~cm}^{-1}\right)$ is considered, where DMSO exhibits several vibrational resonances while D-dodecane is vibrationally nonresonant.

\section{Results and discussion}

Fig. 2a shows the spontaneous Raman and the CARS spectra of pure DMSO in the $\mathrm{CH}_{3}$ stretching region. The two Raman peaks correspond to the symmetric $\left(2914 \mathrm{~cm}^{-1}\right)$ and antisymmetric $\left(2998 \mathrm{~cm}^{-1}\right)$ methyl-stretching vibrations of DMSO. The spectral phase of the CARS emission in this region is depicted in Fig. 2b. For the $2914 \mathrm{~cm}^{-1}$ band, the spectral phase shows the expected $0.5 \pi$ shift at the maximum spectral intensity, and a full $\pi$-shift to the blue side of the resonance. A similar trend is observed for the higher energy band, although the phase shift is somewhat affected by spectral interferences with the lower energy band.

Strong FE-CARS signals are obtained whenever constructive spatial interference alters the lobed CARS emission pattern and introduces radiation along the axial propagation axis. This phenomenon can be clearly seen when analyzing the emission patterns at different axial positions of the focal excitation volume. In Fig. 3a, a representation of the CARS emission pattern close to the propagation axis, obtained by beam scanning the CARS radiation across a small detection aperture, is depicted for various positions along the $z$-scan of the DMSO solution/glass interface. 


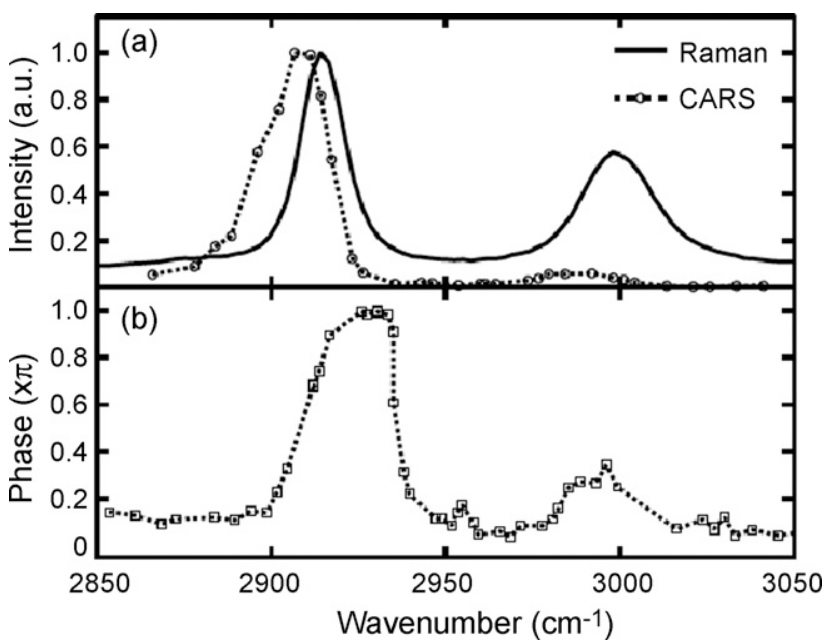

Fig. 2. (a) Raman (solid curve) and CARS (dashed curve with circles) spectra of a mixture of 1:5 DMSO: $\mathrm{H}_{2} \mathrm{O}$ and (b) Spectral phase of the same sample measured using CARS interferometer.

Away from the interface, a quasi-uniform emission pattern is observed. Such a pattern is expected close to the propagation axis, as most CARS radiation is phase-matched in this direction. At the interface, a dip in the CARS signal is obtained at $2933 \mathrm{~cm}^{-1}$. This dip results from the $\pi$-spectral phase shift between the resonant DMSO and the nonresonant glass across the interface, introducing destructive interference between the CARS emission of the two materials. The emission pattern changes slightly, as the CARS radiation is somewhat depleted due to the phase-mismatch in the propagation direction.

A completely different picture is seen in FE-CARS, depicted in Fig. 3b. At depths different from the interface location, an emission pattern is observed that is indicative of the strong phase-mismatch along the propagation axis. The signal is almost completely depleted in the direction of propagation and increases somewhat for larger angles. At the interface, however, we observe strong signals. The spatial extent of the enhanced FE-CARS signal at the longitudinal interface is reminiscent of the axial resolution of the CARS microscope. We attribute this interface sensitivity to the partial cancellation of the engineered destructive interference by

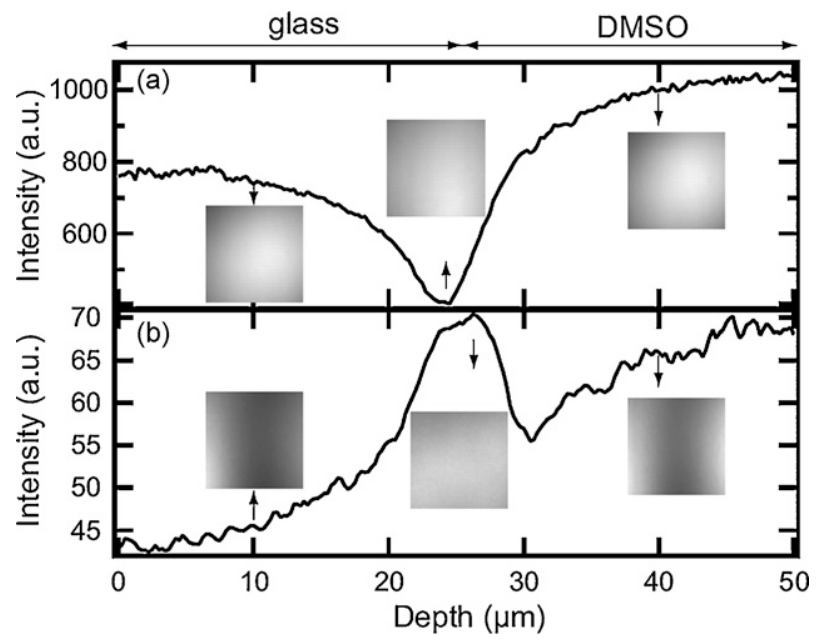

Fig. 3. Depth-scan of glass-DMSO: $\mathrm{H}_{2} \mathrm{O}$ mixture under (a) conventional and (b) $\mathrm{HG} 10$ excitations at $2921 \mathrm{~cm}^{-1}$. The emission patterns at three different depths for each case are shown the plots. Note the change in the emission pattern in (b) near the interface. the spectral phase discontinuity across the interface, introducing phase-matched CARS contributions in the direction of propagation.

Under FE-CARS illumination, differential imaging in the axial direction is observed when the focus is scanned axially across chemical interface. Moreover, differential contrast is brightest at Raman shifts that exhibit a significant spectral phase shift relative to the nonresonant glass signal. This is shown in Fig. 4, which shows that the differential contrast is strongest at $2933 \mathrm{~cm}^{-1}$, where the spectral phase shift is close to $\pi$ (compare with Fig. $2 \mathrm{~b}$ ). When the relative spectral phase shift is small, for instance, at 2869 and $3050 \mathrm{~cm}^{-1}$, the FE-CARS depth-resolved signal follows a similar trend as the conventional CARS signal. The strength of the interfacial signal clearly correlates with the spectral phase of the Raman oscillator, underlining the unique spectroscopic sensitivity of FE-CARS.

Combined with the lateral interface sensitivity previously reported [13,14], the axial differential imaging contrast in FE-CARS allows for object accentuation in multiple dimensions. Fig. 5a shows a FE-CARS image of a layer of DMSO interfaced with a layer of deuterated dodecane, sandwiched between glass slides. At $2933 \mathrm{~cm}^{-1}$, the DMSO layer is moderately vibrationally resonant whereas the D-dodecane layer is far from vibrational resonance. The figure clearly shows the differential imaging contrast in both axial and lateral directions for the resonant DMSO layer. In regular CARS, such contrast is not observed. The lateral and axial crosssections in Fig. 5b and c, respectively, show the strikingly different contrast attained under FE-CARS illumination versus regular CARS excitation.

It is interesting to compare the $\chi^{(3)}$-interface sensitivity of FECARS with the imaging properties of epi-CARS microscopy. Similar to FE-CARS, the epi-CARS technique is sensitive to longitudinal interfaces. Symmetry breaking at the interface lifts the phasemismatch of the backward propagating signal and produces a detectable signal at the epi-CARS detector [22] However, in scattering samples, the epi-CARS signal is typically dominated by signal generated in the phase-matched (forward) direction that has undergone subsequent back scattering. Because the forward CARS signal is many times stronger than the intrinsic epi-CARS response, the signal detected in the backward direction is typically dominated by backward-scattered forward contributions [23]. Hence, in typical biological applications, the unique interface sensitivity of the epi-CARS channel is compromised. In addition, epi-CARS exhibits no differential contrast at interfaces in the lateral dimension. In contrast, FE-CARS provides interface sensitivity in both lateral and longitudinal dimensions, detected through the strong, forward propagating signal.

In this study, we have generated a CARS excitation field with a HG10-type phase pattern through phase shaping of the Stokes beam. It has been pointed out that the reduced overlap of the pump with the focus-engineered Stokes beams leads to less efficient

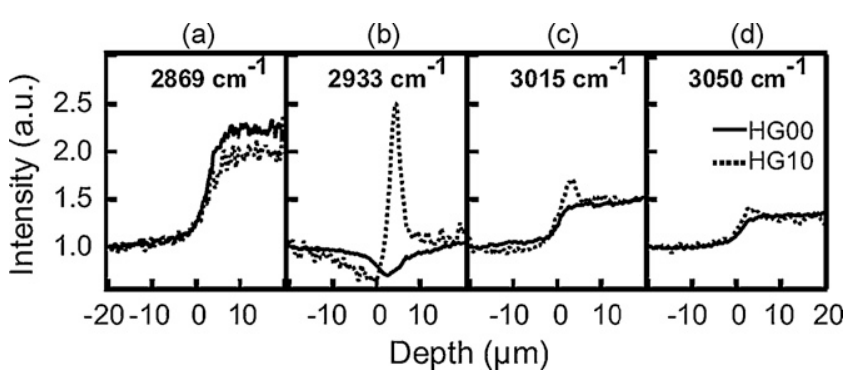

Fig. 4. Spectral dependence of the depth plots of CARS intensity variation near the DMSO: $\mathrm{H}_{2} \mathrm{O}$-glass interface under conventional (solid curve) and focus-engineered (dashed curve) HG10 excitation. 
(a)

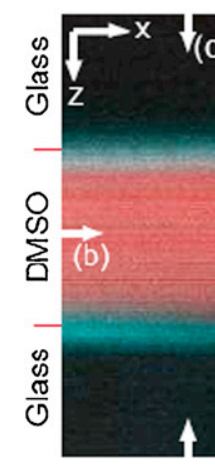

(c)
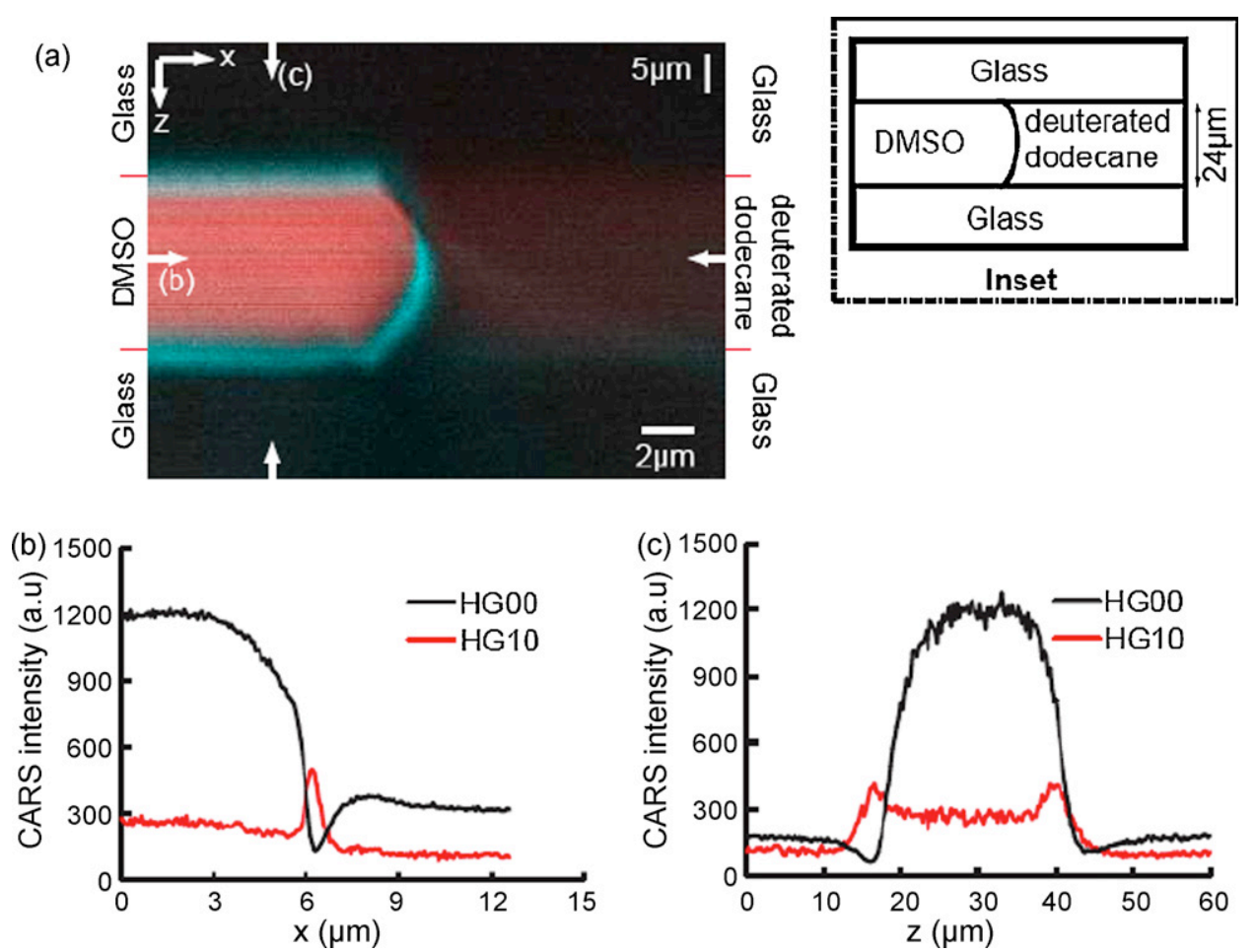

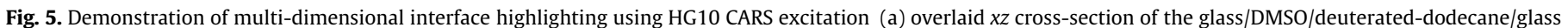

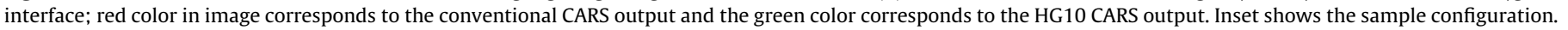

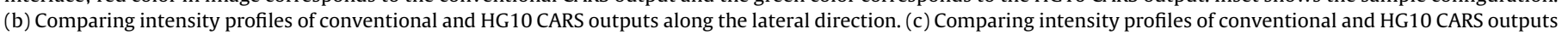
along the longitudinal direction. The location of the profiles plotted in (b) and (c) is indicated with arrows in image (a).

CARS generation [15]. A potential remedy to this problem is found by phase shaping both the pump and Stokes beams [15]. Nonetheless, despite the current non-optimal conditions, vibrationally resonant FE-CARS signals, though weaker, are still within the same order of magnitude as regular CARS signals. It is expected that even better multi-dimensional contrast can be obtained with optimized beam scanning and detection geometries. Our current beam scanner produces a non-stationary beam at the backaperture of the objective lens, introducing non-uniformity across the FE-CARS image. On the detection side, we have used the standard 0.55 NA condenser lens of the microscope. Better suppression of the bulk medium is predicted for a somewhat smaller detection cone angle [13]. With further improvements made to the system, we are confident that high contrast differential vibrational images can be obtained from cell cultures and intact tissues.

\section{Conclusion}

The examples shown in this study illustrate that phasematching control in the CARS focal excitation volume results in interesting new contrast mechanisms. The multi-dimensional differential contrast obtained with HG10 phase profile is particularly interesting for imaging thin biological samples and material films. The ability to accentuate objects with reduced nonresonant background introduces new ways of sample analysis. Importantly, the differential contrast is highly sensitive to the spectral phase and naturally selects vibrationally resonant objects in nonresonant surroundings. This new differential contrast mechanism does not come at the expense of regular CARS contrast, as the spatial light modulator can be swiftly switched between the regular CARS and FE-CARS operation.
We anticipate that the principles of spatial phase dependent emission control will lead to many interesting applications in nonlinear coherent imaging. Applications are not only limited to CARS microscopy, as spatial phase shaping promises novel contrast mechanisms in SHG and THG imaging as well.

We gratefully acknowledge Prof. Ara Apkarian for providing us with the spatial light modulator.

\section{References}

[1] P.J. Campagnola, M. Wei, A. Lewis, L.M. Loew, High-resolution nonlinear optical imaging of live cells by second harmonic generation, Biophys. J. 77 (1999) 33413349.

[2] J.N. Gannaway, C.J.R. Sheppard, Second-harmonic imaging in the scanning optical microscope, Opt. Quant. Electron. 10 (1978) 435-439.

[3] Y. Guo, P.P. Ho, H. Savage, D. Harris, P. Sacks, S. Schantz, F. Liu, N. Zhadin, R.R. Alfano, Second-harmonic tomography of tissues, Opt. Lett. 22 (1997) 1323-1325.

[4] L. Moreaux, O. Sandre, J. Mertz, Membrane imaging by second-harmonic generation microscopy, J. Opt. Soc. Am. B 17 (2000) 1685-1694.

[5] D. Débarre, W. Supatto, E. Farge, B. Moulia, M.-C. Schanne-Klein, E. Beaurepaire, Velocimetric third-harmonic generation microscopy: micrometer-scale quantification of morphogenetic movements in unstained embryos, Opt. Lett. 29 (2004) 2881-2883.

[6] M. Müller, J. Squier, K.R. Wilson, G.J. Brakenhoff, 3D-microscopy of transparent objects using third-harmonic generation, J. Microsc. 191 (1998) 266-274.

[7] J.A. Squier, M. Muller, G.J. Brakenhoff, K.R. Wilson, Third harmonic generation microscopy, Opt. Express 3 (1998) 315-324.

[8] D. Yelin, Y. Silberberg, Laser scanning third-harmonic-generation microscopy in biology, Opt. Express 5 (1999) 169-175.

[9] J.X. Cheng, X.S. Xie, Coherent anti-Stokes Raman scattering microscopy: instrumentation, theory and applications, J. Phys. Chem. B 108 (2004) 827840.

[10] A. Volkmer, Vibrational imaging and microspectroscopies based in coherent antiStokes Raman scattering microscopy, J. Phys. D 38 (2005) R59-R81.

[11] A. Zumbusch, G. Holtom, X.S. Xie, Vibrational microscopy using coherent antiStokes Raman scattering, Phys. Rev. Lett. 82 (1999) 4142-4145.

[12] V.V. Krishnamachari, E.O. Potma, Focus-engineered coherent anti-Stokes Raman scattering: a numerical investigation, J. Opt. Soc. Am. A 24 (2007) 1138-1147. 
[13] V.V. Krishnamachari, E.O. Potma, Imaging chemical interfaces perpendicular to the optical axis with phase-shaped coherent anti-Stokes Raman scattering microscopy, Chem. Phys. 341 (2007) 81-88.

[14] V.V. Krishnamachari, E.O. Potma, Detecting lateral interfaces with focus-engineered coherent anti-Stokes Raman scattering microscopy, J. Raman Spectrosc. 39 (2008) 593-598.

[15] C. Liu, D.Y. Kim, Differential imaging in coherent anti-Stokes Raman scattering microscopy with Laguerre-Gaussian excitation beams, Opt. Express 15 (2007) 10123-10134.

[16] C.L. Evans, E.O. Potma, X.S. Xie, Coherent anti-Stokes Raman scattering interferometry: determination of the real and imaginary components of the nonlinear susceptibility for vibrational microscopy, Opt. Lett. 29 (2004) 2930-2932.

[17] S.H. Lim, A. Caster, S.R. Leone, Single Pulse phase-control interferometric coherent anti-Stokes Raman scattering (CARS) spectroscopy, Phys. Rev. A 72 (2005) 041803.
[18] D.L. Marks, S.A. Boppart, Nonlinear interferometric vibrational imaging, Phys. Rev, Lett. 92 (2004), 123901:1-123901:4.

[19] D. Oron, N. Dudovich, Y. Silberberg, Femtosecond phase-and-polarization control for background-free coherent anti-Stokes Raman spectroscopy, Phys. Rev. Lett. 90 (2003), 213905:1-213905:4.

[20] E.O. Potma, C.L. Evans, X.S. Xie, Heterodyne coherent anti-Stokes Raman scattering (CARS) imaging, Opt. Lett. 31 (2006) 241-243.

[21] L. Novotny, B. Hecht, Principles of Nano-optics, Cambridge University Press, New York, 2006.

[22] J.-X. Cheng, A. Volkmer, X.S. Xie, Theoretical and experimental characterization of coherent anti-Stokes Raman scattering microscopy, J. Opt. Soc. Am. B 19 (2002) $1363-1375$.

[23] C.L. Evans, E.O. Potma, M. Puoris'haag, D. Côte, C. Lin, X.S. Xie, Chemical imaging of tissue in vivo with video-rate coherent anti-Stokes Raman scattering microscopy, Proc. Natl. Acad. Sci. U.S.A. 102 (2005) 16807-16812. 